Supporting Information for cg060814c

\title{
Structure Variation of Mercury(II) Halide Complexes with Different Imidazole-Containing Ligands
}

Xiao-Feng Wang, ${ }^{\dagger}$ Yang Lv, ${ }^{\dagger}$ Taka-aki Okamura, ${ }^{\ddagger}$ Hiroyuki Kawaguchi, ${ }^{\S}$ Gang Wu, ${ }^{\dagger}$ Wei-Yin Sun, ${ }^{*}{ }^{\dagger}$ and Norikazu Ueyama ${ }^{\ddagger}$

Coordination Chemistry Institute, State Key Laboratory of Coordination Chemistry, School of Chemistry and Chemical Engineering, Nanjing University, Nanjing 210093, China, Department of Macromolecular Science, Graduate School of Science, Osaka University, Toyonaka, Osaka 560-0043, Japan, and Coordination Chemistry Laboratories, Institute for Molecular Science, Okazaki, Aichi 444-8585, Japan

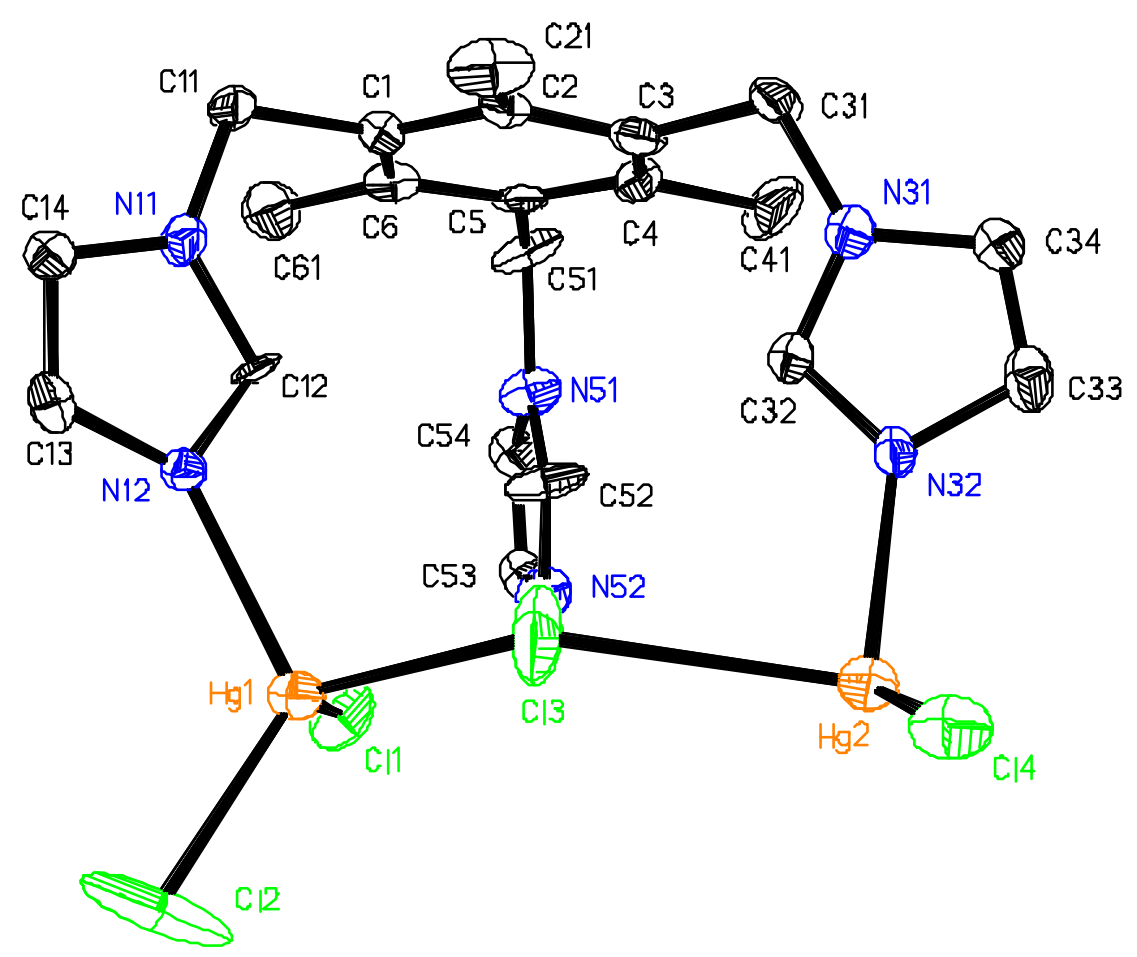

Figure S1. X-ray crystal structure of 7 with the atom numbering scheme (ellipsoids at $30 \%$ probability). 


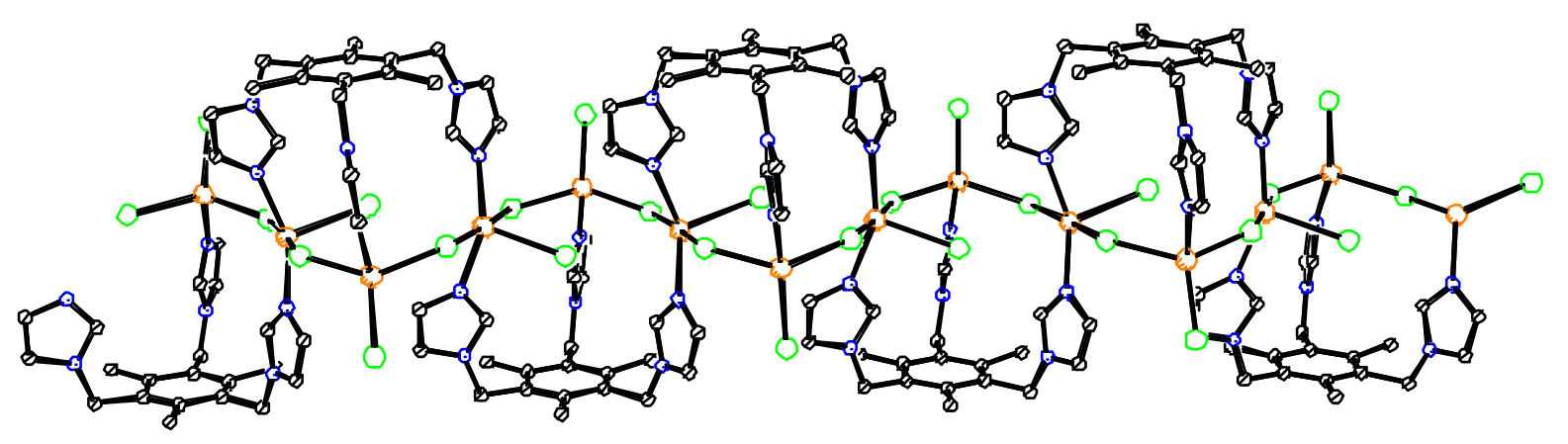

Figure S2. A view of 1D inorganic $\mathrm{Hg}-\mathrm{Cl}-\mathrm{Hg}$ chain of 7.

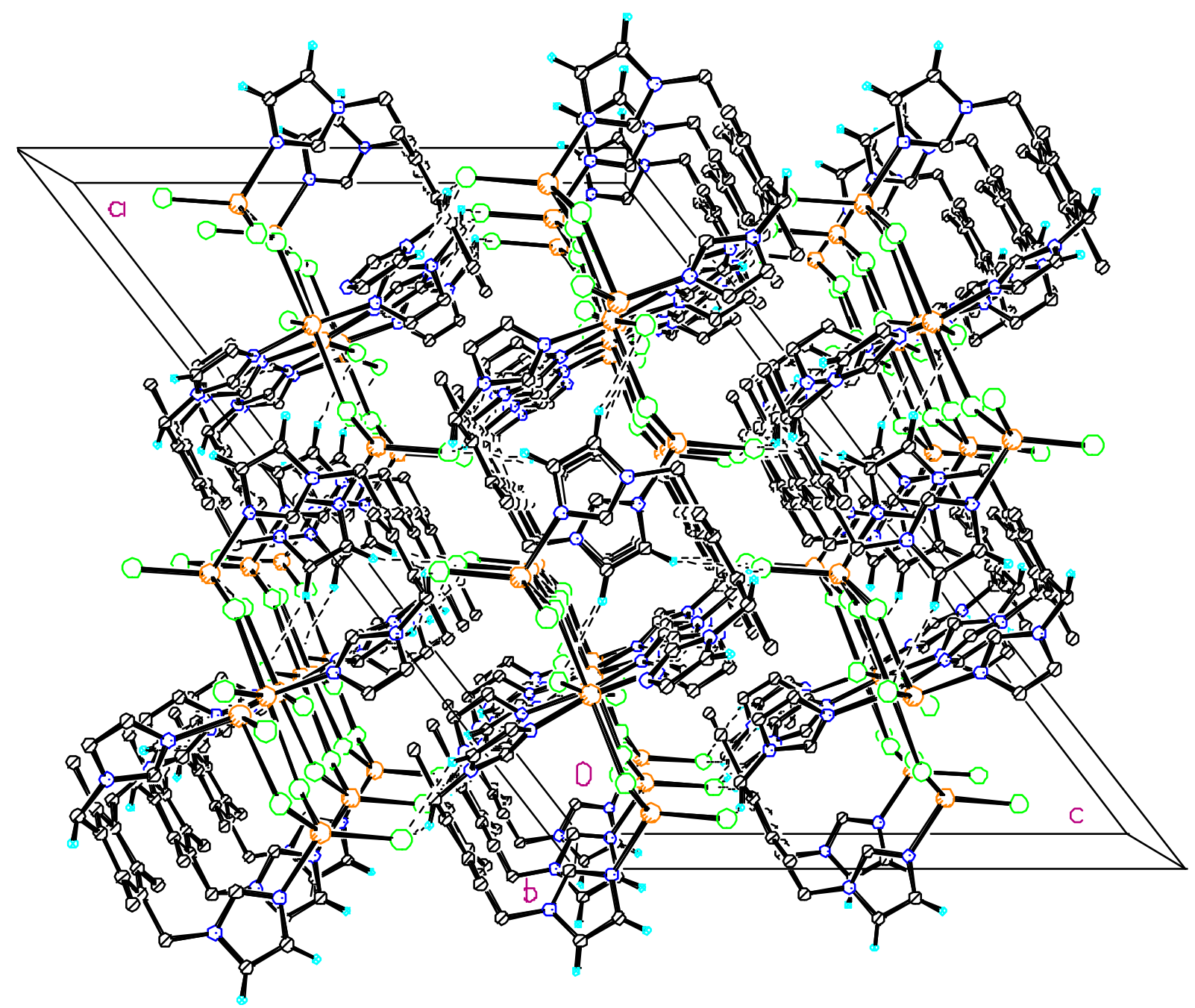

Figure S3. Crystal packing diagram of complex 7 with the hydrogen bonds indicated by dashed lines. 